\title{
MORPHOLOGY-BASED BUILDING DETECTION FROM AIRBORNE LIDAR DATA
}

\author{
Xuelian Meng, Research Assistant \\ Nate Currit, Assistant Professor \\ Geography Department, Texas State University-San Marcos \\ 601 University Drive, San Marcos, TX 78666 \\ xm1001@txstate.edu \\ currit@txstate.edu \\ Le Wang, Assistant Professor \\ Department of Geography, State University of New York at Buffalo \\ Wilkeson Quad, Buffalo, NY 14261 \\ lewang@buffalo.edu
}

\begin{abstract}
The advent of Light Detection and Ranging (LIDAR) technique provides a promising resource for three-dimensional building detection. Most current methods commonly fuse LIDAR data with other multi-spectral images to help remove vegetation based on NDVI or other vegetation indices; however, the fusing process may cause errors that are introduced by resolution differences, geo-referencing, time differences, shadow and high-rise building displacement problems. Due to the difficulty of removing vegetation, relatively few approaches have been developed to detect buildings only from LIDAR data. This paper presents a morphological building detection method to identify buildings by gradually removing non-building pixels. First, a ground filtering algorithm separates ground from buildings, trees, and other objects. Then an analytical approach further removes the remaining non-building pixels using size, shape, height, building element structure, and height difference between the first and last return. The experiment results show this method provides a comparative performance with an overall accuracy of $95.46 \%$ as in the study site in the Austin urban area.
\end{abstract}

\section{INTRODUCTION}

Automatic building detection from high spatial-resolution remote sensing images has gained wide attention for GIS data production, three-dimensional urban visualization, urban intra-structure analysis, and hazard damage evaluation (Cho et al., 2004; Peng and Liu, 2005; Peng et al., 2005). Especially traditional urban land-cover and land-use analysis is mainly based on pixels instead of urban elements. The success of building detection not only allows urban researchers to analyze urban phenomena based on urban elements but also to gain rich elementassociated information by simulating the human ability to differentiate elements from photographs (Jensen, 2000).

Building detection requires high-resolution aerial photographs or satellite images with resolution higher than 5 meters, which can express buildings as objects instead of mixed pixels (Jensen, 2000; Jin and Davis, 2005). Traditional color or multi-spectral images commonly present shadows and high-rise building displacement problems and make building detection troublesome. The newly-developed LIDAR technique measures the actual threedimensional coordinates of the reflectance from ground objects instead of a snap-shot from an angular view, hence it is free of the influence of sun shine and high-rise building displacement problems with an extra building-height bonus (Zhang et al., 2006). Furthermore, the unique difference between the first and last return in vegetation area helps to remove most vegetation. On the other hand, some regularly shaped objects appear as similar shapes to buildings and are hard to determine by visual interpretation. Researchers can easily solve this problem by automatic building detection from LIDAR data. Since this research chooses LIDAR as the main resource, the following review will focus on LIDAR-related building detection.

Many techniques have been developed to detect buildings from LIDAR data, and the most popular approach is to fuse LIDAR data with multi-spectral images. Popular approaches include image segmentation (Rottensteiner and Briese, 2003; Cho et al., 2004), morphology (Cho et al., 2004), classification (Forlani et al., 2006), and methods based on vegetation indices (Sohn and Dowman, 2003, 2007; Rottensteiner et al., 2003). Relatively few methods have been developed to identify buildings only from LIDAR data because of the difficulty to fully remove vegetation (Zhang et al., 2006; Morgan and Tempfli 2000). Developing comparative techniques to identify buildings

ASPRS 2008 Annual Conference

Portland, Oregon • April 28 - May 2, 2008 
only from LIDAR data is worthwhile because fusing LIDAR data with other resources will introduce errors into building detection. First, fusing multi-resource data with different resolutions adds errors to building candidates. In order to obtain NDVI, the most frequently used multi-spectral images are IKONOS with a resolution of 4 meters, while airborne LIDAR data for building detection can usually reach 0.5-1 meters (Sohn and Dowman, 2003). The fusing process usually produces a resolution in between, which sacrifices LIDAR resolution (Tullis and Jensen, 2003). Secondly, the fusing process, such as registration and resampling, introduces errors in building detection. On the other hand, building or mountain shadows, clouds, and high-rise building displacement problems due to the sensors' angular view may cause errors in building detection. These errors counteract the advantage of fusing LIDAR data with other resources, and bring the final building accuracy down. Third, the cost and availability of other high-resolution images is critical for data producing in industry. The purpose of this research is to develop an alternative building-detection method only based on LIDAR data. Users can easily adapt this method with other resources when extra images are available.

\section{MORPHOLOGY BASED BUILDING DETECTION}

Researchers derive buildings either by identifying buildings directly or by gradually removing non-building objects, such as ground and vegetation. This method first removes ground using a ground-filtering algorithm, and then further removes the remaining non-building pixels using size, shape, height, building element structure, and the height difference between the first and last return based on a morphology-based analytical approach, as shown in Fig. 1. The major procedures can be grouped into two steps: ground filtering and remaining non-building removing.

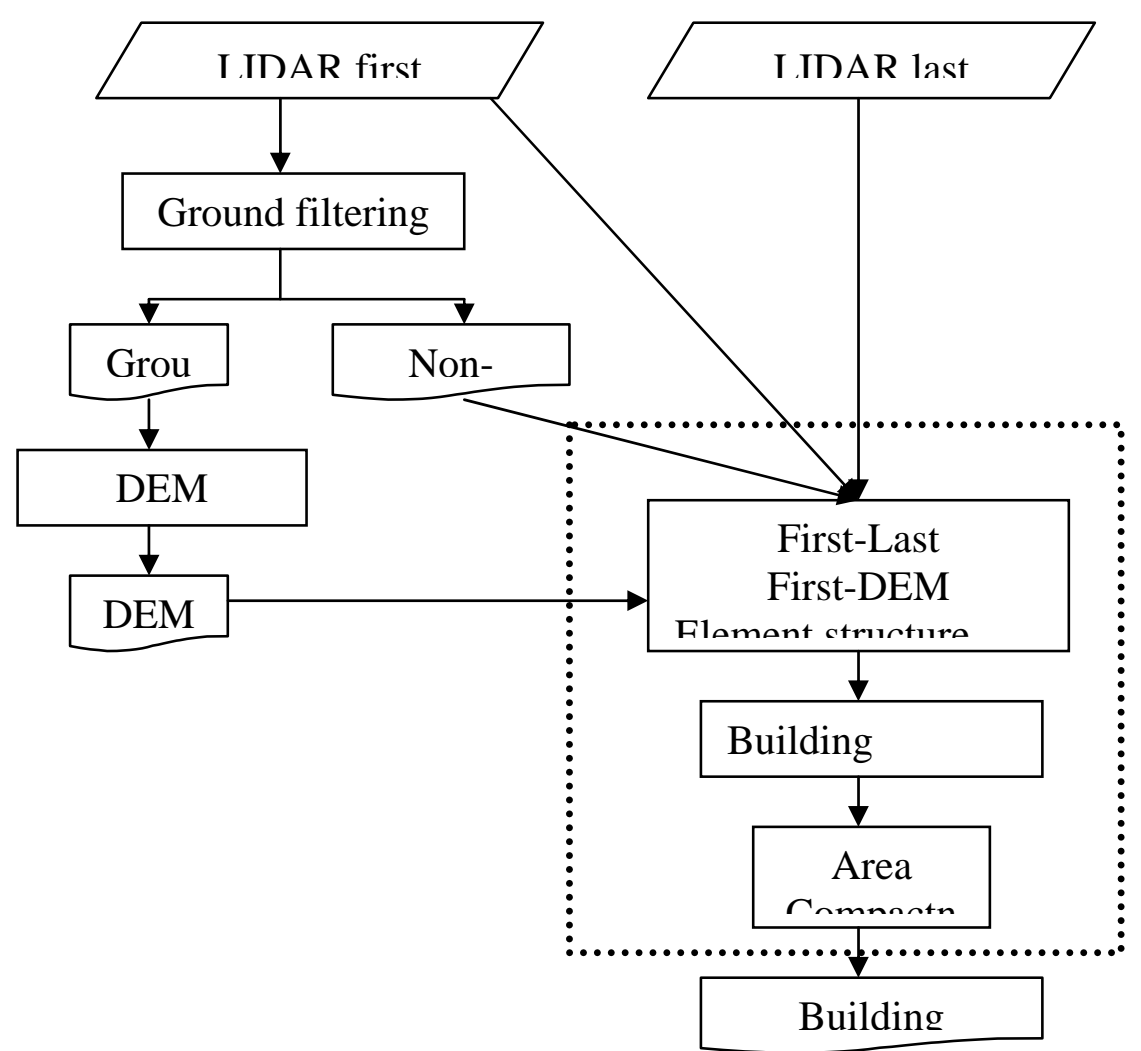

Figure 1. Flow chart of the morphology-based building detection. 


\title{
Ground Filtering
}

Researchers have proven that ground filtering is effective and critical to remove non-building objects (Morgan and Tempfli, 2000; Zhang et al., 2006). This research applies a multi-directional ground filtering algorithm on the gridded LIDAR first return to identify ground and generates a Digital Elevation Model (DEM) based on the results (Meng, 2005). This filter provides sound performance in dense urban areas with complex ground covering, such as trees, shrubs, short walls, and vehicles. The raw LIDAR data of the first and last returns are irregularly distributed point clouds. A two-dimensional mesh is overlayed on the point clouds, and the elevation of the nearest point is assigned to the elevation of the cell. Then the ground filter gradually removes non-ground based on thresholds of slopes, elevation difference between the point and its local minimum elevation, and the elevation difference between the point and the nearest ground point. Users can choose other alternative methods to fit study sites if necessary. After ground filtering, this research generates a DEM at the same resolution based on the detected ground pixels to calculate object heights.

\section{Removing Non-building Pixels Based on Morphology Operators}

After removing ground pixels, the remaining pixels are mainly composed of vegetation, buildings, and other objects such as shrubs, short walls, and vehicles. Buildings differ from other objects in size, shape, height, and texture by visual interpretation in LIDAR data. Besides, the elevation difference between the first and last return and the building element structure are considerable factors to remove vegetation. This research carefully arranged the differentiating factors in an order illustrated in Fig. 1 to maximize the effect in each procedure. Specifically, this method first utilizes three pixel-based operations to break non-building blocks into smaller fragments. A building recovering process refills holes in buildings for pixels wrongly removed in the previous process so as to refine buildings before converting to vector objects. Finally, this research converts building candidates into vectors, and then an object-based area and compactness analysis further removes non-building parcels based on thresholds. The following section gives a detailed introduction about those procedures in the dash frame in Fig. 1.

The large elevation difference between the first and last returns is a critical indicator for tall vegetation, and height differentiates buildings with low non-building objects. The active LIDAR sensors send out laser beams to ground and accept multiple returns depending on the distances the laser beams penetrate. The first return records the reflectance from ground and object surfaces, and the last return documents the deepest points the laser beams reach. Laser lights possess a relatively strong ability to penetrate vegetations and hardly pass hard man-made objects, such as building roofs. Theoretically, the elevation differences among ground or building areas should be 0; however, these elevation differences commonly present small values in practice due to LIDAR measurement accuracy and data preprocess. As a result, this research applies an elevation difference threshold to filter out vegetation with higher differences. On the other hand, object heights can derive by the difference between the first return and the bare earth model DEM. This method removes objects that are shorter than the minimum building height from the building candidates, such as short walls, shrubs, and vehicles.

Some tree fragments in dense tree areas may have similar size and morphological characteristics, which makes them difficult to remove. But dense trees or trees attached to buildings present more irregular linear shapes on boundaries than buildings. Hence, this research designed a building element structure operator to break dense vegetation into smaller fragments and detach remaining linear trees from buildings. Building structure in neighboring pixels may differ depending on spatial resolutions. The following illustration applies to images with resolutions equal or higher than one meter, which is often the case for urban LIDAR data. In a high resolution image, buildings are not supposed to be linear lines since buildings are usually wider than 1 meter. Given a three by three neighborhood as in figure 2, if both the pixels above and below the pixel $(\mathrm{i}, \mathrm{j}$ ) are non-buildings, or both the pixels on the left and right side of the pixel $(i, j)$ are non-buildings, then the center pixel $(i, j)$ is removed from building candidates. Although some buildings have irregular sharp angles and few pixels on the corners of the sharp angles may be wrongly removed, this operation is worthwhile because it effectively breaks down vegetation fragments and prevents more errors of labeling trees as buildings.

\author{
ASPRS 2008 Annual Conference \\ Portland, Oregon • April 28 - May 2, 2008
}



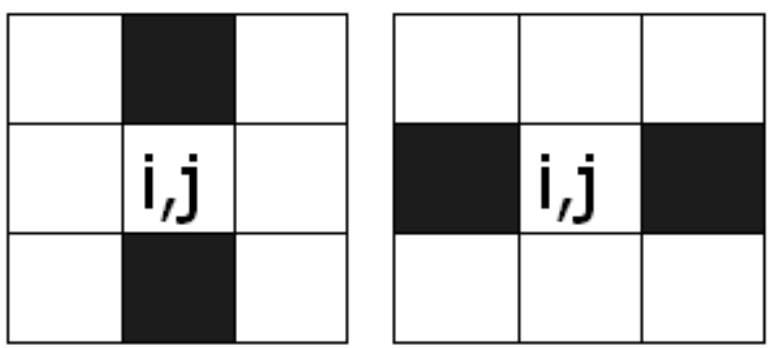

Figure 2. Building elementary structure filtering.

Unlike most methods, this research designed a building recovering process to fill the holes in buildings caused by previous processes before converting building candidate pixels to objects. In the results of elevation difference filtering between the first and last returns, some pixels within building polygons may be wrongly removed due to the sensor errors, preprocesses, or thin objects on top of roofs such as power lines, antennae, and vegetation. Experiments prove that these building holes increase the difficulty to separate buildings with vegetation based on morphology indices, such as roundness or compactness. These noisy pixels in buildings usually randomly appear within buildings and usually contain few pixels. Given a nine by nine neighborhood, a vegetation fragment is likely to have more than one non-building pixel. For each non-building pixel, this filter compares the elevation of the pixel with the average value of its neighboring building candidate pixels. If the elevation difference is lower than the threshold, then the pixel is added to the building candidate. Results prove that the recovering process effectively refills holes in buildings but has little influence on vegetation fragments. Finally, this research converted building candidate pixels into vectors and further removed non-building parcels based on area and compactness thresholds. Equation 1 illustrates the compactness calculation. The output based on object analysis is the final results for building candidates.

Compactness $=$ area $\times$ peremeter $^{2} /(4 \uparrow)$

\section{STUDY SITE}

The study site is located on the campus of the University of Texas at Austin, and covers an area of $0.67 \mathrm{~km}^{2}$. The site is on a hillside covered by dense urban features, such as bridges, vehicles, trees, flagpoles, fences, shrubs, short walls, and buildings with various sizes and shapes. An Optech ALTM LIDAR sensor collected the raw LIDAR data in the year 2000. A resampling process based on the nearest principle converts the first- and last-return point clouds to images with a 0.5-meter resolution. Figure 3 shows the grided first-return image of study site, and the red polygons are building ground truth data that is modified from the building vector data in 2003 based on a field trip on March 24, 2007.

\section{RESULTS AND DISCUSSION}

This research applied a 0.2-meter threshold for the elevation difference between the first and last return, a 2meter height threshold to remove off-terrain objects lower than the minimum building height, and a $40-\mathrm{m}^{2}$ area threshold to remove objects smaller than the minimum building size. Figure 4 is the result of the final building candidates overplayed on top of the first return. The accuracy assessment shows $95.46 \%$ of the overall accuracy, and a 0.89 Kappa value. The overall accuracy shows the percentage of the points that are correctly identified, which is obtained by dividing the number of correctly identified ground and non-ground points with the total number of points. Kappa value considers the performance in different classes based on confusion matrix (Jensen, 2005). Figure 5 is the classified error spatial distribution analysis. Black and white pixels are points that are correctly identified. The blue color represents the error of labeling building as non-building, and the red color demonstrates the error of labeling non-building as building. The results show that this method successfully identified most buildings. Few buildings that are smaller than the minimum building threshold are removed from the building candidates, which are

ASPRS 2008 Annual Conference

Portland, Oregon A April 28 - May 2, 2008 
mainly for equipment or storage. Some larger dense vegetation parcels are troublesome to remove as the vectors present similar size and compactness.

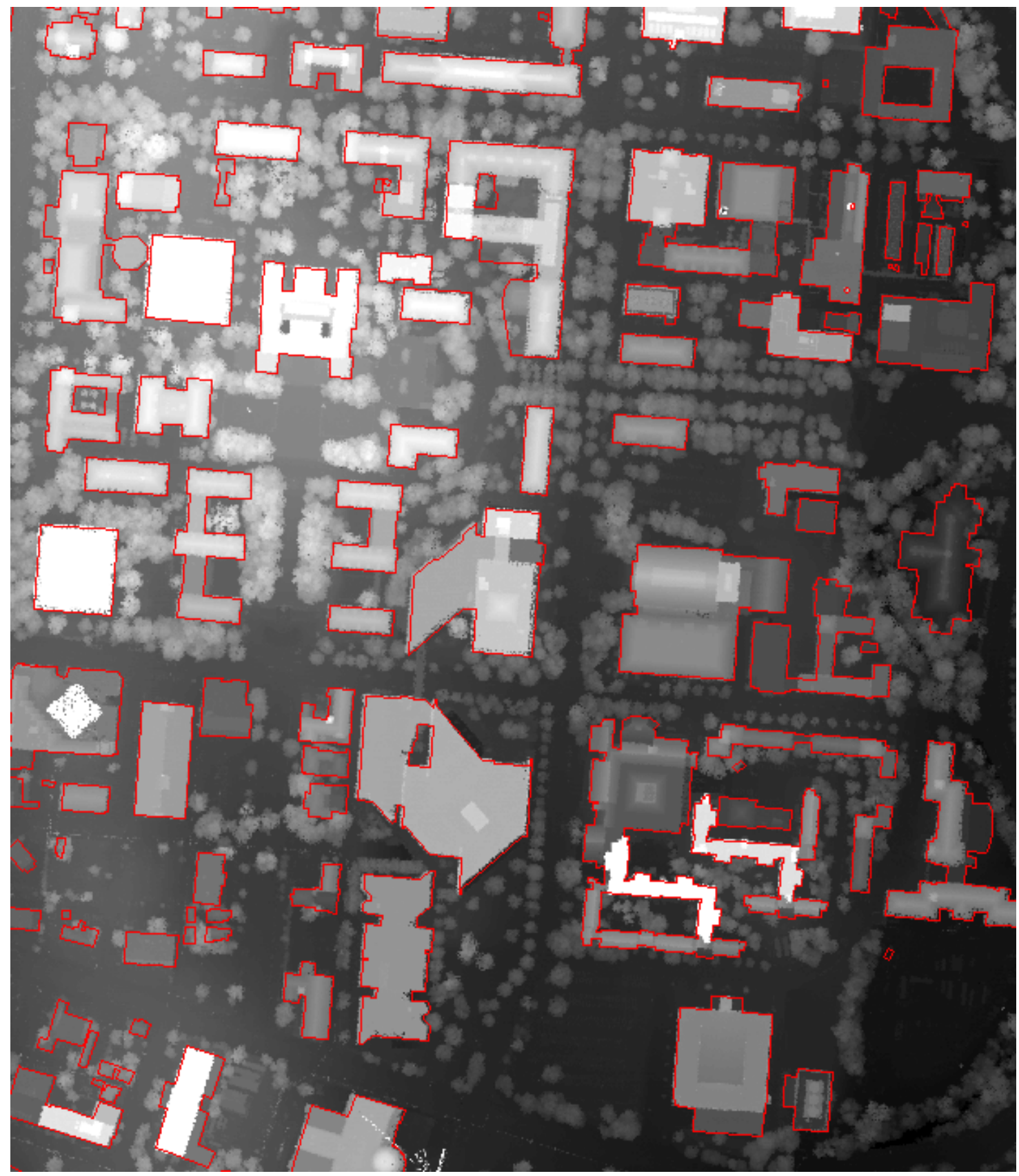

Figure 3. The LIDAR first return and building ground truth data. 


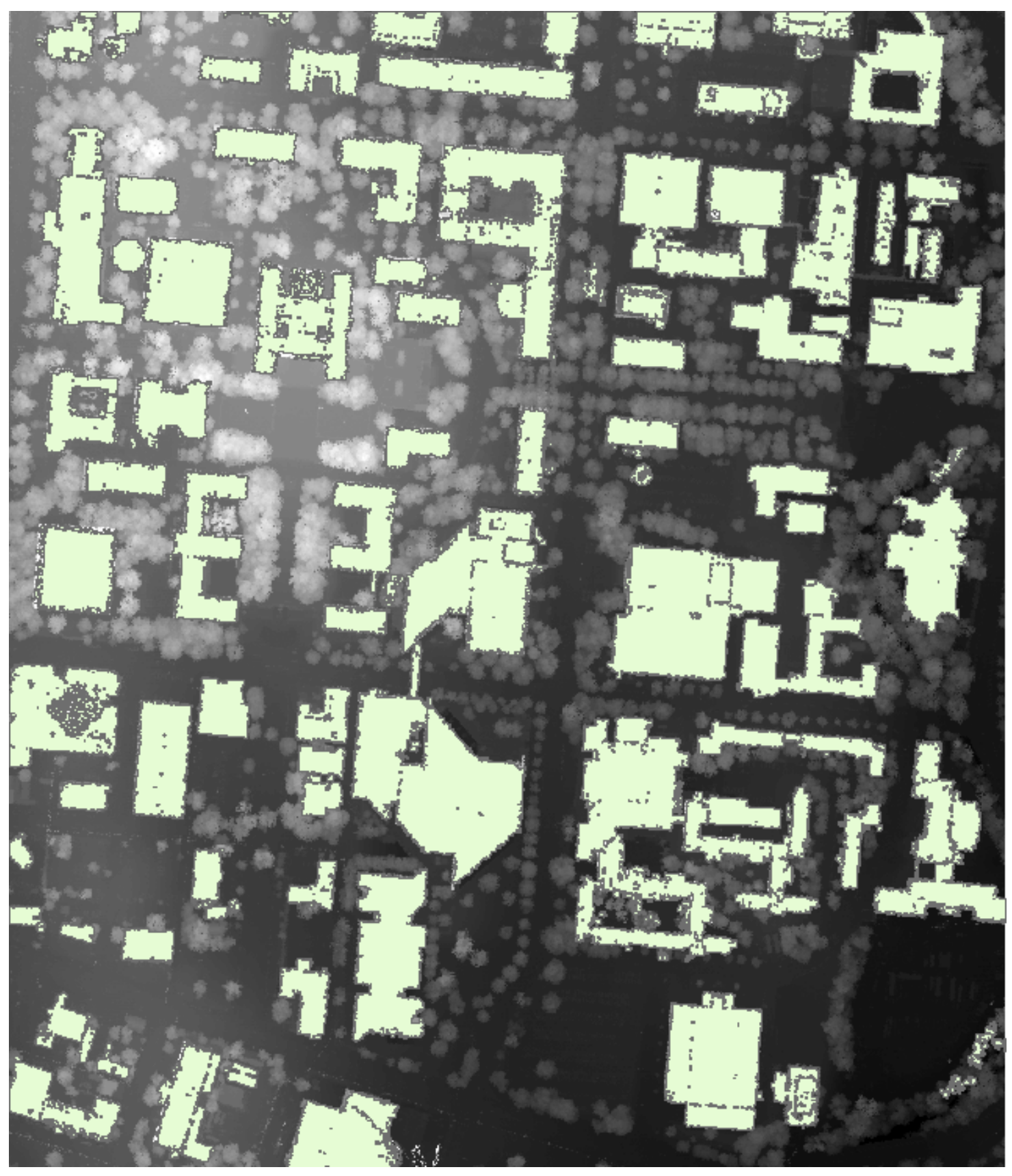

Figure 4. Building detection results. 


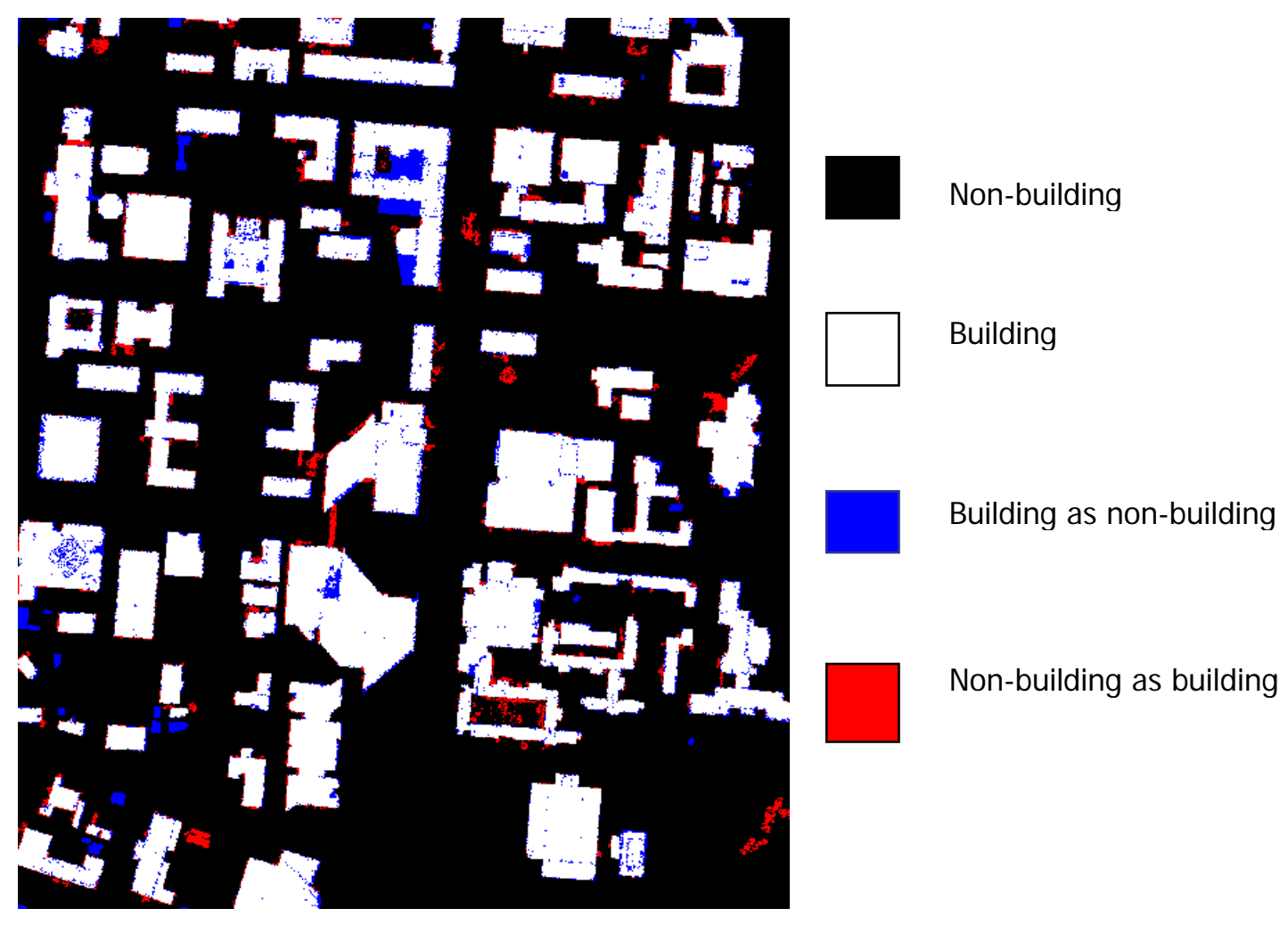

Figure 5. Error spatial distribution analysis for building detection. Black and white pixels are points correctly identified. The blue color represents the error of labeling building as non-building, and the red color demonstrates the error of labeling non-building as building.

To further analyze the major factors that lead to the $4.54 \%$ errors, this research examines the major blue and red error fragments by referencing the high-resolution Google Earth images as in figure 6. As a result, the first error component includes several small buildings that are smaller than the minimum building size threshold. This is because, when selecting the minimum building size, researchers usually think about the residential or commercial buildings. While in this study site, most of the buildings being removed are mall buildings for storage, green houses, outside equipment, and other functions. As an example, (A) in figure 6 is a fitness storage room. The second error component originates from errors in ground truth data or the building definition difference between the algorithms and the ground truth data. As shown in (B) of Fig. 6, there are three error clusters labeling building as non-building. The upper cluster is a concrete surface with the same height as the ground, but is higher than the underground building. Similarly, drop ground within the buildings in Fig. 6(D) and (F) are surfaces with the same heights as the outside ground surfaces, but are wrongly digitized as buildings. The lower cluster in Fig. 6(B) is a concrete surface for an open dining area, which is elevated around 30 centimeters above ground. Not able to view buildings in threedimension, the digitizers for building vectors are more likely to identify the surface as a part of buildings. This means that the ground truth data and the building detection algorithms may have different definitions about buildings for some complex structures. The third error component is due to the sensor errors, preprocesses, or thin objects on top of roofs such as power lines, antennae, and vegetation. Fig. 6(C) is a high rise building with many small antennae or sensors on the top of the building, which cause the error in the process of the LIDAR first and last return elevation diffidence filtering. Fig. 6(B) is a roof with a south edge as low as the brushes and ground, which may be identified as ground in the ground filtering process. The fourth error type is caused by dense vegetation that is hard to penetrate by laser beams. Fig. 6(E) is an example of a dense tree area that is difficult to remove. Overall, this research tested the morphological building detection method in a developed urban area with complicated building structures. 


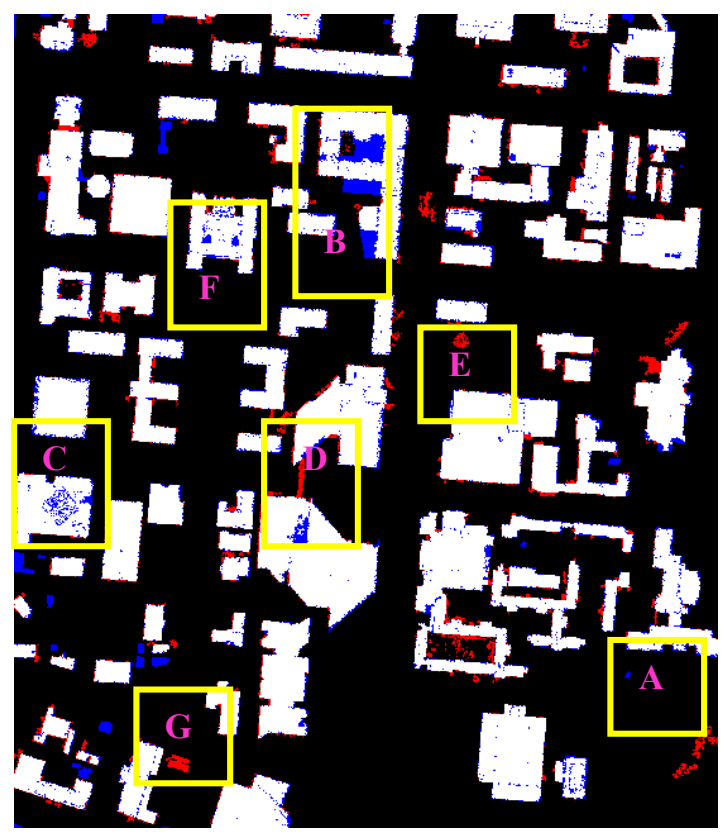

Figure 6. Building detection error analysis by referencing Google Earth images.
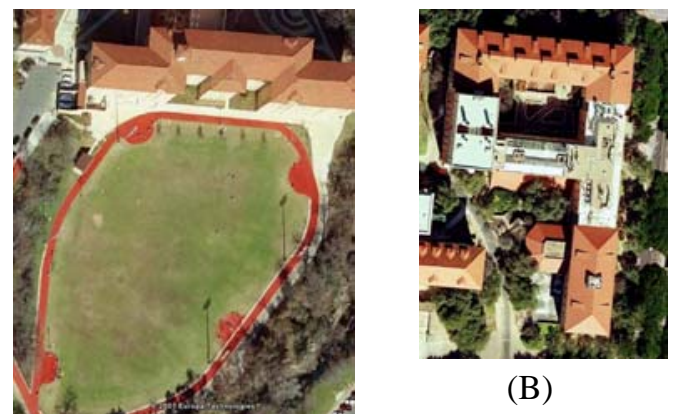

(B)

(A)

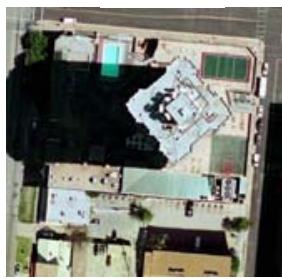

(C)

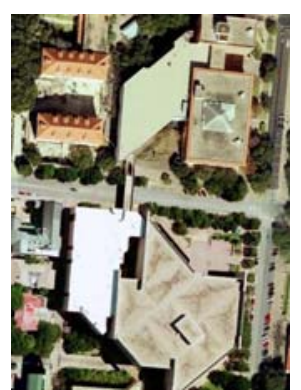

(D)

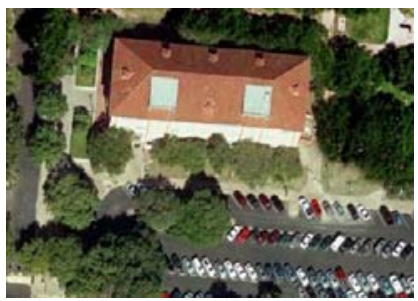

(E)

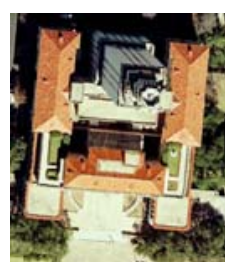

(F)

\section{CONCLUSION}

The advent of the LIDAR technique provides a promising resource to detect three-dimensional buildings. Current LIDAR-based methods commonly work on a combination of LIDAR and other multi-spectral images due to the difficulty of removing vegetation, and relatively few methods only apply on LIDAR data. Using multi-resource images introduces errors during the fusing process due to preprocess, and resolution or time differences. This research developed an alternative morphological building-detection method based on LIDAR data by gradually removing non-building pixels. The experiment results show that this method provides a comparative performance with an overall accuracy of $95.46 \%$ as in a study site in the Austin urban area.

\section{REFERENCES}

Cho, W., Jwa, Y., Chang, H., \& Lee, S., 2004. Pseudo-grid based building extraction using airborne LIDAR data, ISPRS Commission III, Geo-Imagery Bridging Continents, XXth ISPRS Congress.

Forlani, G., Nardinocchi, C., Scaioni, M., \& Zingaretti, P., 2006. Complete classification of raw LIDAR data and 3D reconstruction of buildings, Pattern Anal Applic, 8: 357-374.

Jensen, J.R., 2005. Introductory Digital Image Processing: a Remote Sensing Perspective. Prentice Hall: New York. 
Jin, X. \& Davis, C.H., 2005. Automated building extraction from high-resolution satellite imagery in urban areas using structural, contextual, and spectral information, EURASIP Journal on Applied Signal Processing, 2005(14): 2196-2206.

Meng, X., 2005. A slope- and elevation-based filter to remove non-ground measurements from airborne LIDAR Data, UCGIS Summer Assembly, June 28-July 1, Jakson Hole, Wyoming-USA. URL: Http://www.ucgis.org/ summer2005/studentpapers/meng.pdf, Accessed February, 2008.

Morgan, M., \& Tempfli, K., 2000. Automatic building extraction from airborne laser scanning data, In Proc. $19^{\text {th }}$ ISPRS Congr., Amsterdam-The Netherlands, book 3B, pp. 616-623.

Peng, J. \& Liu, Y., 2005. Model and context-driven building extraction in dense urban aerial images, International Journal of Remote Sensing, 26(7): 1289-1307.

Peng, J., Zhang, D., \& Liu, Y., 2005. An improved snake model for building detection from urban aerial images, Pattern Recognition Letters, 26(5): 587-595.

Rottensteiner, F. \& Briese, C., 2003. Automatic generation of building models from LIDAR data and the integration of aerial images, ISPRS, Vol. XXXIV, Dresden.

Rottensteiner, F., Trinder, J., Clode, S., \& Kubik, K., 2003. Building detection using LIDAR data and multi-spectral images, Proc. VIIth Digital Image Computing: Techniques and Applications, Sydney-Australia, pp.10-12.

Sohn, G. \& Dowman, I., 2003. Building Extraction. Using LIDAR DEMs and IKONOS Images, ISPRS, Volume XXXIV, PART 3/W13. Dresden-Germany, pp. 8-10.

Sohn, G. \& Dowman, I., 2007. Data fusion of high-resolution satellite imagery and LiDAR data for automatic building extraction, ISPRS Journal of Photogrammetry and Remote Sensing, 62(1): $43-63$.

Tullis, J. A. \& Jensen, J.R., 2003. Expert system house detection in high spatial resolution imagery using size, shape, and context, Geocarto International, 18(1): 5-15.

Zhang, K., Yan, J., \& Chen, S., 2006. Automatic construction of building footprints from airborne LIDAR data, IEEE Transactions on Geoscience and Remote Sensing, 44 (9): 2523-2533. 